\title{
Rabaska
}

Revue d'ethnologie de l'Amérique française

\section{Cartographie des terrains d'enquête de Marius Barbeau dans Charlevoix (1916-1940)}

\section{Christian Harvey}

Volume 13, 2015

Présence de Marius Barbeau : l'invention du terrain en Amérique

française. Autour d'un legs centenaire (1914-2014)

URI : https://id.erudit.org/iderudit/1033746ar

DOI : https://doi.org/10.7202/1033746ar

Aller au sommaire du numéro

Éditeur(s)

Société québécoise d'ethnologie

ISSN

1703-7433 (imprimé)

1916-7350 (numérique)

Découvrir la revue

Citer cet article

Harvey, C. (2015). Cartographie des terrains d'enquête de Marius Barbeau dans Charlevoix (1916-1940). Rabaska, 13, 31-36. https://doi.org/10.7202/1033746ar d'utilisation que vous pouvez consulter en ligne. 


\section{Cartographie des terrains d'enquête de Marius Barbeau dans Charlevoix (1916-1940) \\ Christian Harvey \\ Société d'histoire de Charlevoix Centre de recherche sur l'histoire et le patrimoine de Charlevoix}

Jusqu'à aujourd'hui, les études consacrées à Marius Barbeau dans le domaine des traditions orales ont presque exclusivement porté sur l'analyse du matériel recueilli lors de ses enquêtes plutôt que sur sa méthode d'approche du terrain ${ }^{1}$. Cette omission, volontaire ou non, a malheureusement laissé dans l'ombre son apport scientifique majeur dans le développement de la recherche ethnologique au Québec et au Canada français. Et surtout, elle a offert un champ libre à des commentateurs comme Gérard Bouchard venant jeter un certain discrédit sur une démarche portant, selon eux, «peu d'attention [...] aux ruptures, aux redéfinitions, à la production de nouvelles formes culturelles. ${ }^{2}{ } \gg$

En 2004, l'étude doctorale de Serge Gauthier ${ }^{3}$ a révélé des données surprenantes, à tout le moins aux yeux de l'observateur contemporain, sur la localisation des informateurs rencontrés par Marius Barbeau dans la région de Charlevoix. Contre une certaine approche spontanée du terrain, Gauthier a permis d'établir que l'ethnologue a pour les fins de son travail constitué de véritables lieux de folklore dans l'espace régional qui furent par la suite, avec des changements mineurs, fréquentés notamment par Luc Lacourcière et Félix-Antoine Savard. L'ethnologue Marius Barbeau a donc construit, le plus souvent d'une manière implicite, mais aussi dans des notes de cours

1. Serge Gauthier, Charlevoix ou la création d'une région folklorique. Étude du discours de folkloristes québécois (1916-1980), Québec, Presses de l’Université Laval, 2006, XIII-208 p.

2. Gérard Bouchard. "L'Historiographie du Québec rural et la problématique nord-américaine avant la Révolution tranquille. Étude d'un refus ", Revue d'histoire de l'Amérique française, vol. 44, no 1, été 1990, p. 206.

3. Serge Gauthier, «Charlevoix ou la création d`une région folklorique : étude du discours de folkloristes québécois (1916-1980) », Thèse de Ph. D. (Ethnologie historique), Université Laval, 2004, $235 \mathrm{p}$. 


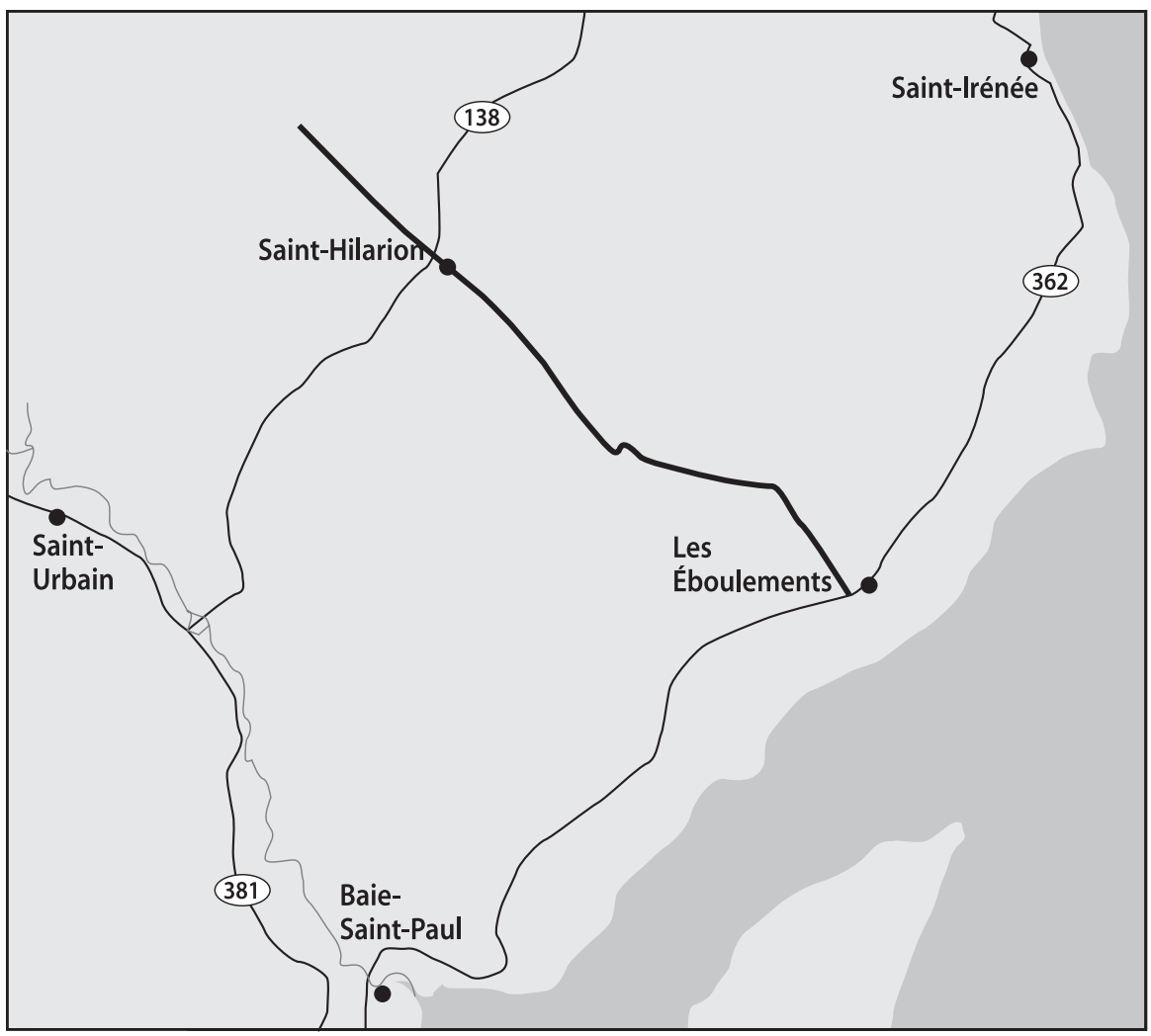

Carte 1

La route du folklore selon les enquêtes de Marius Barbeau en Charlevoix (1916-1941).

remises à ses étudiants ${ }^{4}$, une véritable approche méthodologique du terrain d'enquête différente de celle du simple amateur.

" Dis-moi comment tu cherches je te dirai ce que tu cherches" ${ }^{5}$ ), rappelait justement le philosophe Ludwig Wittgenstein. En fait, l'analyse de la méthode de Marius Barbeau nous ramène finalement à la question bien plus fondamentale de tout travail d'un chercheur : qu'est-ce que je cherche au juste ? Une étape essentielle afin «d'objectiver le sujet de l'objectivation $^{6} \gg$ selon les mots du sociologue Pierre Bourdieu. Cela peut nous amener aujourd'hui à donner un sens nouveau à l'œuvre protéiforme de Marius Barbeau et à ouvrir de nouvelles pistes de recherche.

4. Marius Barbeau, « En quête de connaissances anthropologiques et folkloriques dans l'Amérique du Nord depuis 1911 », Québec, Archives de folklore, 1945.

5. Ludwig Wittgenstein, Remarques philosophiques, Paris, Gallimard, 1975, p. 66.

6. Voir Pierre Bourdieu, Raisons pratiques sur la théorie de l'action, Paris, Seuil, 1994, 247 p. 


\section{Les résultats}

D'abord, nous devons présenter quelques remarques concernant les sources et la méthode de compilation utilisées par Serge Gauthier. Son dépouillement a été réalisé grâce à la consultation des fiches de présentation des enregistrements sonores réalisés par Marius Barbeau entre 1916 et 1941 dans Charlevoix, des documents conservés aux Archives de l'Université Laval. Chaque pièce indiquait le nom de l'informateur, son lieu de résidence et souvent même le nom du rang où il habitait. De l'ensemble, seulement les informateurs provenant de Charlevoix ont été retenus. Marius Barbeau a donc rencontré au cours de cette période d'un quart de siècle 93 informateurs différents (tableau 1).

\section{Tableau 1}

Provenance des informateurs de folklore dans Charlevoix

Collection Marius Barbeau (1916-1941)

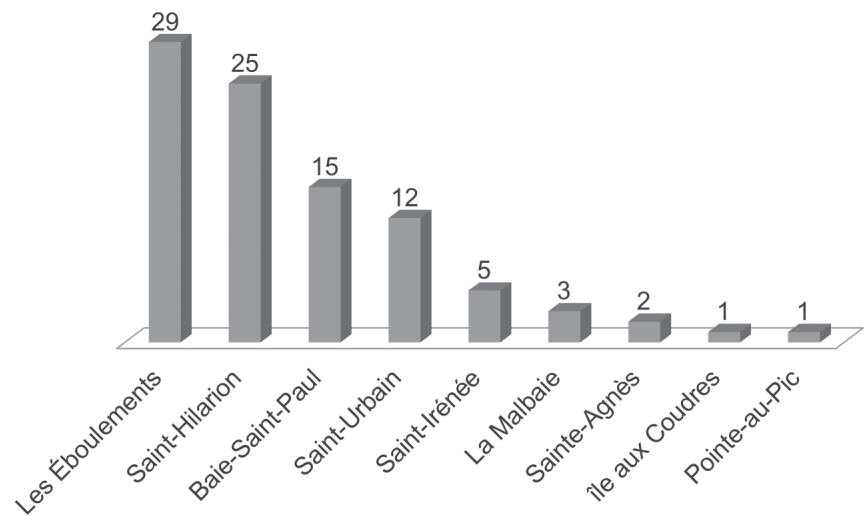

À première vue, on peut noter que les informateurs proviennent majoritairement de la partie ouest de la région, plus spécifiquement du plateau intermédiaire s'élevant entre les vallées du Gouffre, dans le secteur de Baie-Saint-Paul, et la vallée de la rivière Malbaie, dans le secteur de La Malbaie.

En consultant le tableau 2, on peut observer que les informateurs proviennent à $87 \%$ de quatre municipalités : Les Éboulements, Saint-Hilarion, Baie-Saint-Paul et Saint-Urbain, dont 58 \% pour Les Éboulements et SaintHilarion seulement (carte 1). Pourquoi donc une telle concentration spatiale? Comme le note Serge Gauthier, il est difficile de voir en cela le résultat « de circonstances hasardeuses », mais plutôt « un choix délibéré, structuré ${ }^{7}$.

Avant d'aller plus loin, quelques informations complémentaires doivent être mentionnées. Les données fournissant le nom du rang de l'informateur

7. Serge Gauthier, Charlevoix ou la création d'une région folklorique, op. cit., p. 135. 


\section{Tableau 2}

\section{Provenance des informateurs de folklore dans Charlevoix}

Collection Marius Barbeau (1916-1941)

Résultats en pourcentage

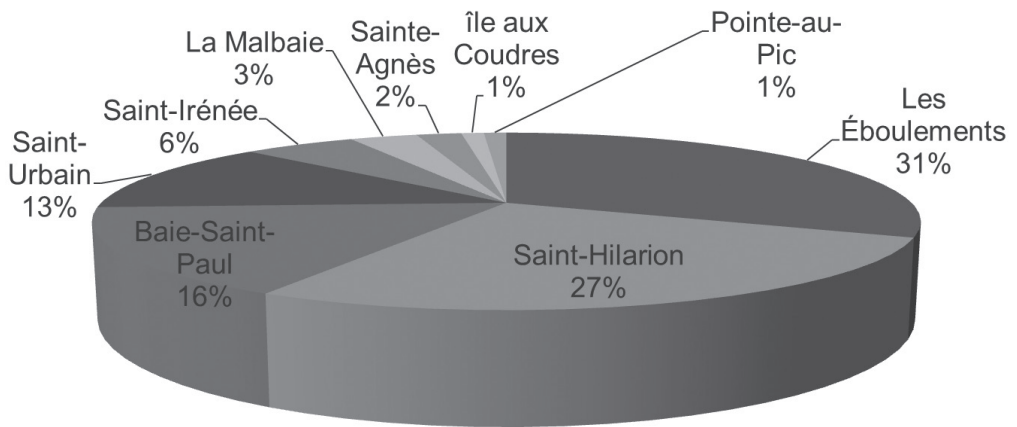

viennent réduire la limite spatiale retenue par Marius Barbeau. Seulement deux informateurs proviennent du village de Baie-Saint-Paul. Les autres proviennent majoritairement des rangs Saint-Ours et Sainte-Croix. À SaintHilarion, la principale mention est celle du rang Chiguère où se rend Barbeau à partir des années 1920. Aux Éboulements, ce sont les rangs Sainte-Marie et Saint-Joseph qui font l'objet des enquêtes.

Pour synthétiser, la « route du folklore » selon Marius Barbeau dans la région de Charlevoix se trouve donc concentrée le long ou autour d'un chemin reliant le village des Éboulements au rang Saint-Antoine ou Chiguère de Saint-Hilarion. Ce primat de Saint-Hilarion et des Éboulements devient encore plus dominant avec les Luc Lacourcière et Félix Antoine Savard (tableau 3): 111 informateurs sur 312, soit $36 \%$, pour Saint-Hilarion ; 59 informateurs sur 312, soit $19 \%$ pour Les Éboulements. Si l'on additionne les enquêtes de Marius Barbeau et de Lacourcière/Savard (tableau 4), on arrive à un total de 136 sur 405, soit $34 \%$, pour Saint-Hilarion. Les Éboulements suivent avec 88 sur 405 , soit $22 \%$. 


\section{Tableau 3}

\section{Provenance des informateurs de folklore dans Charlevoix}

Collection Marius Barbeau (1916-1941)

Pourcentage Lacourcière/Savard

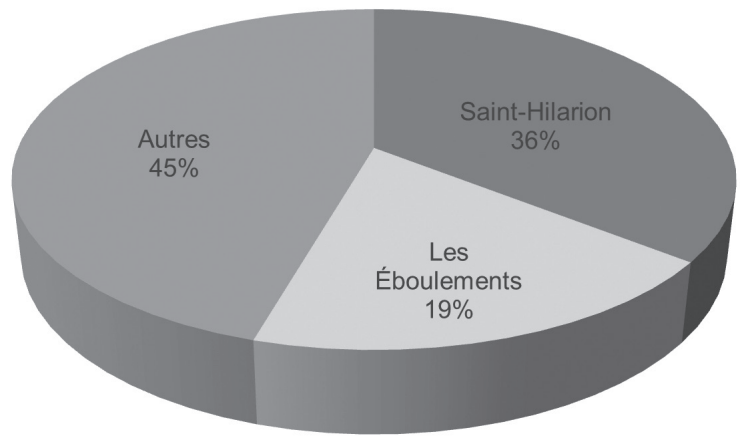

Tableau 4

Provenance des informateurs de folklore dans Charlevoix

Collection Marius Barbeau (1916-1941)

Pourcentage global

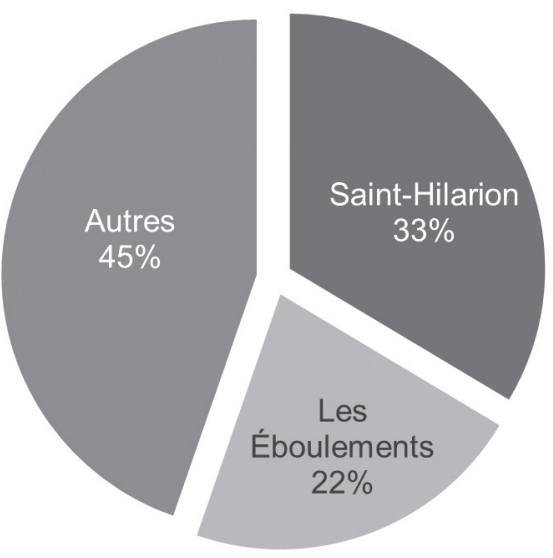

\section{Caractéristiques des lieux de folklore}

En général, Marius Barbeau a retenu des localités possédant des caractéristiques communes : population plutôt limitée, faiblesse de la présence de notables, économie composée d'un mélange d'agriculture et d'exploitation forestière, éloignement des institutions scolaires supérieures. D'une manière un peu schématique, afin de maintenir la pureté de son enquête, Marius 
Barbeau a cherché un informateur peu lettré, voire analphabète, vivant dans un rang plutôt qu'un village, peu en contact (du moins selon sa connaissance) avec le monde extérieur et particulièrement avec la villégiature. L'arrièrepays occupe donc une place disproportionnée par rapport à son importance économique, sociale et démographique.

Marius Barbeau ne recherchait pas une représentativité de la population globale de Charlevoix, ni les influences autochtones, écossaises ou irlandaises sur la culture locale, mais un milieu propice à la conservation d'une tradition orale issue de la France ancienne apportée au XVII ${ }^{\mathrm{e}}$ siècle et maintenue en vie depuis en terre d'Amérique. Il a donc établi une méthode bien précise si bien que la compilation ne peut être le simple effet du hasard.

\section{De la méthode au point de vue}

Marius Barbeau a donc élaboré d'une manière implicite et explicite, dans son enquête, une méthode d'approche du terrain qui l'a amené à créer des lieux de folklore dans Charlevoix. L'exercice aurait sans doute pu être réalisé ailleurs, dans un autre terroir de l'Amérique française. Il privilégie la conservation des traditions et pratiques venues de la France chez des Canadiens français. Une analyse actuelle devrait donc se questionner sur ce rôle conjoint de la reproduction et de la transformation des pratiques issues de la France d'Ancien régime dans la production de la culture québécoise et de la francophonie nord-américaine. Aujourd'hui, dans une perspective multiculturelle, on semble ne plus tenir compte de cette reproduction culturelle et les recherches en ce domaine en milieu universitaire semblent s'effectuer presque exclusivement hors du Québec. Espérons que ce colloque, se déroulant dans Charlevoix, puisse permettre de retrouver les traces du chemin parcouru par Marius Barbeau, ce pionnier dans la construction d'une méthode scientifique en ethnologie. 